\title{
BMJ Open Improving discharge care: the potential of a new organisational intervention to improve discharge after hospitalisation for acute stroke, a controlled before- after pilot study
} Dominique A Cadilhac, ${ }^{1,2}$ Nadine E Andrew, ${ }^{1}$ Enna Stroil Salama, ${ }^{3}$ Kelvin Hill, ${ }^{4}$
Sandy Middleton, ${ }^{5}$ Eleanor Horton, ${ }^{6}$ lan Meade, ${ }^{7}$ Sarah Kuhle, ${ }^{8}$ Mark R Nelson, ${ }^{9}$
Rohan Grimley, ${ }^{10,11}$ On behalf of the Australian Stroke Clinical Registry Consortium

\section{ABSTRACT}

Andrew NE, Stroil Salama E, et al. Improving discharge care: the potential of a new organisational intervention to improve discharge after hospitalisation for acute stroke, a controlled beforeafter pilot study. BMJ Open 2017;7:e016010. doi:10.1136/ bmjopen-2017-016010

- Prepublication history and additional material are available. To view these files please visit the journal online (http://dx.doi org/10.1136/bmjopen-2017016010).

Received 19 January 2017 Revised 22 May 2017 Accepted 14 June 2017

\section{CrossMark}

For numbered affiliations see end of article.

\section{Correspondence to} A/Prof Dominique A Cadilhac; dominique.cadilhac@monash. edu
Objective Provision of a discharge care plan and prevention therapies is often suboptimal. Our objective was to design and pilot test an interdisciplinary, organisational intervention to improve discharge care using stroke as the case study using a mixed-methods, controlled before-after observational study design.

Setting Acute care public hospitals in Queensland, Australia $(n=15)$. The 15 hospitals were ranked against a benchmark based on a composite outcome of three discharge care processes. Clinicians from a 'top-ranked' hospital participated in a focus group to elicit their success factors. Two pilot hospitals then participated in the organisational intervention that was designed with experts and consumers.

Participants Hospital clinicians involved in discharge care for stroke and patients admitted with acute stroke or transient ischaemic attack.

Intervention A four-stage, multifaceted organisational intervention that included data reviews, education and facilitated action planning.

Primary and secondary outcome measures Three discharge processes collected in Queensland hospitals within the Australian Stroke Clinical Registry were used to select study hospitals: (1) discharge care plan; (2) antihypertensive medication prescription and (3) antiplatelet medication prescription (ischaemic events only). Primary measure: composite outcome. Secondary measures: individual adherence changes for each discharge process; sensitivity analyses. The performance outcomes were compared 3 months before the intervention (preintervention), 3 months postintervention and at 12 months (sustainability).

Results Data from 1289 episodes of care from the two pilot hospitals were analysed. Improvements from preintervention adherence were: antiplatelet therapy (88\%vs96\%, $p=0.02$ ); antihypertensive prescription (61\%vs $79 \%, p<0.001)$; discharge planning (72\%vs $94 \%$, $\mathrm{p}<0.001)$; composite outcome $(73 \% \mathrm{vs} 89 \%, \mathrm{p}<0.001)$. There was an insignificant decay effect over the 12-month sustainability period (composite outcome: $89 \%$ postintervention vs $85 \%$ sustainability period, $p=0.08$ ).

\section{Strengths and limitations of this study}

- Hospitals offer the ideal environment to instigate individualised prevention management strategies for chronic conditions with a high risk of recurrence, such as stroke. However, few effective interventions are available to improve recommended and highquality discharge care. A strength of this research is that is has begun to fill this important knowledgepractice gap.

- The organisational intervention was carefully designed using theory, evidence, involvement of consumers and a broad range of clinical and implementation sciences experts.

- Standardised and routinely collected data from a national clinical quality registry were used to ensure reliable comparisons over time, including ability to measure the long-term sustainability impact, the new frontier in quality improvement.

- The effectiveness data ( $n=1289$ episodes of care) were obtained from two hospitals and trialling in a larger number of hospitals to confirm the findings is needed.

- Similar to other non-randomised studies, there is the potential for reporting and response bias.

Conclusion Discharge care in hospitals may be effectively improved and sustained through a staged and peerinformed, organisational intervention. The intervention warrants further application and trialling on a larger scale.

\section{INTRODUCTION}

In terms of the total global burden of disease, neurological disorders combined with cerebrovascular disease represents $7.1 \%$ for all causes and ages. ${ }^{1}$ Stroke contributes to the majority of this burden, and similar to other chronic neurological conditions, patients are hospitalised for acute treatment. A hospital 
provides the ideal environment in which to instigate individualised prevention management strategies for chronic neurological conditions with a high risk of recurrence, such as stroke where the cumulative 10-year risk is about $40 \% .^{23}$

Because of the sudden impact of chronic disability that may be a consequence of stroke, comprehensive discharge planning, that involves the patient and family, is recommended to facilitate transition back into the community. The objective of interdisciplinary and formalised discharge planning is to reduce length of stay, improve the coordination of services following discharge and avoid readmission and long-term unmet needs. ${ }^{45}$ Unfortunately, evidence from national stroke audits and the Australian Stroke Clinical Registry (AuSCR) consistently show that less than three in five patients receive a discharge care plan or behavioural prevention education from clinicians, and prescription of prevention medication is suboptimal. ${ }^{6} 7$ This is despite the availability of standardised tools to help clinicians undertake these activities more efficiently in Australia such as the Stroke Foundation's 'My Stroke Journey' discharge pack that comprises an education booklet and care planning guide. ${ }^{8}$ A better understanding of clinician and hospital factors related to discharge care is needed to support the development of more effective interventions that target clinician practice to ensure optimal discharge care. ${ }^{910}$

The aims of this study were to design and pilot test an interdisciplinary, organisational intervention to improve discharge care using stroke as the case study. In particular, we sought to determine the preliminary effectiveness of the new intervention using routinely collected data from the AuSCR by comparing preimplementation and postimplementation adherence results (combined as a composite outcome and for individual processes of care) and the change in performance relative to benchmarks established for these processes across the different measurement periods (ie, referred to here as a change in performance gap score). We also sought to measure sustainability of any improvements over a 12-month time period, as sustainability outcomes are rarely reported. ${ }^{11-13}$ The primary outcome was the net change in the overall composite outcome and secondary outcomes were changes in individual scores and the gap score. Further details are provided below.

\section{METHODS}

The description and reporting of our intervention is based on the Standards for QUality Improvement Reporting Excellence 2.0 guidelines to promote completeness of information, transparency and potential for replication in the field. ${ }^{14}$

\section{Context}

Acute care public hospitals located in Queensland and using the AuSCR for performance monitoring were the setting for the intervention. Discharge care involves different health professionals. ${ }^{5}$ In acute hospitals, where there is a high turnover of beds and short lengths of stay (average for stroke is about 5 days), ${ }^{7}$ omissions may occur. Designing interventions for achieving behaviour change can be challenging when health professionals are time poor or where roles and responsibilities for different aspects of care are shared. Furthermore, it remains unclear if multifaceted organisational interventions are more effective than single-component interventions or whether there is a dose-response relationship. ${ }^{15}$

\section{Intervention development and design}

This was a mixed-methods study that was iteratively undertaken whereby results of each stage were reviewed to inform the next stage. ${ }^{16}$ The basis of the intervention was to use external facilitation to raise awareness of areas of underperformance with local clinicians and workup strategies to leverage enabling factors and reduce barriers to successful implementation of the discharge processes relevant to the local context. The intervention development and pilot testing occurred over a 5-month period. A summary of each phase is provided below with additional detail provided in the online Supplemental methods.

Preintervention design phase: understanding what works well at 'top-performing' hospitals

We included a preintervention design phase whereby two hospitals with the greatest adherence to the discharge processes were selected to provide information on the factors that made provision of discharge care successful at their site. These were defined as 'top-performing' or 'exemplar' hospitals. Qualitative data from these exemplar hospitals were subjected to thematic analysis and the factors that were found to influence adherence to the clinical care processes were mapped across the 14 domains of the theoretical domains framework (TDF). ${ }^{18}$ This provided a conceptual basis for describing the enablers and barriers to implementation.

\section{Intervention design phase}

The intervention was designed using complementary knowledge translation concepts and theory including elements of the 'Promoting Action on Research Implementation in Health Services' (PARIHS) framework, transtheoretical model of change; the plan-do-studyact method and the Ottawa model of research use. ${ }^{919-22}$ In an attempt to fast track strategy development at the pilot hospitals, successful techniques reported from the 'exemplar' hospitals were shared (online supplemental results, table I). An expert working group $(n=9)$ that included decision-makers, clinicians, experts in knowledge translation and consumer representatives finalised the intervention with the project team via one face-to-face workshop and two teleconferences.

The final intervention, known as STELAR (Shared Team Efforts Leading to Adherence Results), was delivered in four discrete stages: pre-workshop survey (to gain a better understanding of current discharge processes and practices); two facilitated workshops (workshop 1: to discuss aspects of existing practices and gather consensus on the potential 


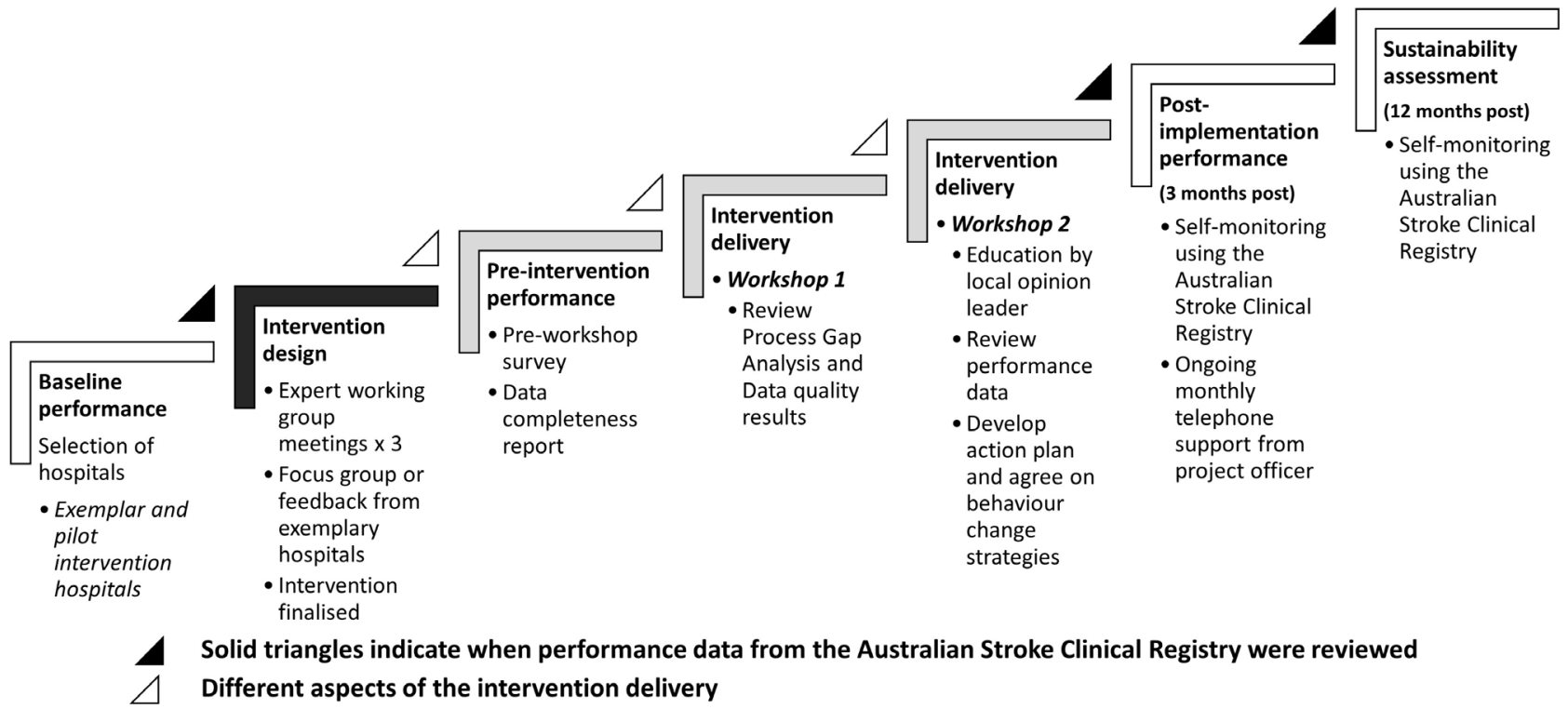

Figure 1 Project stages and evaluation periods.

barriers that could be modified; workshop 2: an education component delivered by a 'local opinion leader' followed by a review of local AuSCR performance data by the external facilitator (DAC) and then development of a local action plan and appointment of a local 'change champion' who would facilitate implementation of the agreed strategies. The strategies aimed to maximise use of existing resources and processes, rather than increasing staff workloads) and ongoing telephone/electronic support by the programme team for 2 months after the second workshop. During this time, retraining in AuSCR data collection processes was also provided, as required. (figure 1 and online supplemental methods, figure I). A staged approach was used to improve knowledge of capability, motivation and opportunity within the settings, which have been recognised as fundamental to understanding the targeted behaviours. ${ }^{23}$ In brief, the delivery mechanisms included external facilitation; performance feedback with a gap analysis and a codesigned educational meeting using a local opinion leader, evidence from exemplar hospitals and a structured action planning activity. Participants were hospital staff involved in delivering the discharge processes including medical, nursing, pharmacy and allied health clinicians to be identified by the local opinion leader. The majority of these delivery mechanisms were informed by the literature. ${ }^{171924}$

\section{Measures of intervention effectiveness}

Hospital performance was measured using patient-level data entered into the AuSCR by clinicians at participating hospitals using a standardised approach. ${ }^{25}$ In brief, all patients admitted to hospital with stroke or transient ischaemic attack (TIA) are eligible to be included in this national clinical quality registry and an opt-out procedure is used, or waiver of consent for people who die in hospital, to reduce selection bias. Clinical performance data collected in relation to discharge care as part of the AuSCR in Queensland are: (1) provision of a discharge care plan developed with the interdisciplinary team and the patient and/or family; (2) prescribed antihypertensive medication at discharge from hospital or (3) if an ischaemic stroke or TIA, prescribed antiplatelet/anticoagulant medications at discharge from hospital. ${ }^{7}$ For a full description refer to online supplementary table II .

The primary effectiveness outcome used in this study was a composite score of adherence to each of the three discharge processes combined from both hospitals (referred to as the overall composite outcome). Adherences to individual processes were also evaluated, as was adherence relative to benchmarks for each of the processes and the composite outcome (referred to as performance ' $\mathrm{gap}$ ' score) (see the Data analysis section and online supplementary figure II). Results from individual hospitals and in combination are presented.

\section{Selection of hospitals}

Public hospitals in Queensland that had registered at least 20 patients in the AuSCR between January 2012 and June 2013 were eligible $(n=15 ; 82 \%)$. The two exemplar hospitals were chosen from sites that fell within the top 20th percentile for the composite gap score.

To pilot test this new intervention, we selected two hospitals that would benefit from improvement in adherence to these care processes (referred to here as 'pilot hospitals') 
and these were chosen from sites that fell within the bottom 20th percentile (online supplemental methods, figure III). One and half years of data were used to ensure that we identified sites with consistently high or low levels of performance. We also interrogated the data from the selected pilot sites to ensure that their results were not an artefact of missing or poorly entered data. As more than two hospitals met the criteria, hospital selection was also based on: relative performance across the individual care processes; the extent to which the hospital was representative of other similar hospitals to improve the generalisability of the results and ability to participate within our project time frame. Stroke unit medical directors of the eligible hospitals were sent an invitation letter in January 2014 and then consent to participate was obtained.

\section{Ethical considerations}

The project was approved by the Metro North Hospital and Health Service, The Prince Charles Hospital Human Research Ethics Committee (HREC/13/QPCH/279, November 2013) and governance approval was obtained from each participating hospital. Approval for the project was also granted by the AuSCR Research Task Group and governance committees.

\section{Data analysis}

Assessment of hospital performance for discharge care

Adherence of each hospital to the care processes was calculated as the number of patients who received the discharge process divided by the number of patients who were eligible for the care process. The composite outcome was calculated as the total number of patients who received the three discharge processes divided by the total number of patients eligible to receive the three care processes. Benchmarks were also calculated for each care process, based on a modified version of the Achievable Benchmarks of Care approach ${ }^{26}$ previously used in stroke by Hall et $a l,{ }^{27}$ whereby the benchmark is calculated using at least $15 \%$ of the data.

The benchmarks were then used to calculate a gap score for each hospital for each discharge process and the composite outcome. To calculate this, adherence scores were divided by the relevant benchmark to provide the gap score (eg, if a site had an adherence score of $40 \%$ and the benchmark was $80 \%$, the gap score would be $50 \%$ as they would be halfway towards meeting the benchmark) (online supplementary figure II provides an overview of the equations used). Separate benchmarks were calculated, using data from all 15 hospitals, for deriving the gap scores for each evaluation period. This approach accounted for the potential influence of other quality improvement activities being undertaken in Queensland between the different time periods used in the analyses and secular trends, as well as reducing issues of ceiling effects associated with individual care processes.

\section{Changes in performance over time}

Analysis of patient-level AuSCR data was used to determine the effectiveness of the intervention applying a historically controlled before-after design comparing the composite outcome scores. Time periods compared were: 3 months prior to the commencement of the intervention (preintervention period January to March 2014); 3 months after workshop 2 (postintervention period, October to December 2014) and 12 months after the postintervention period to assess if the programme had sustained effects (sustainability period, October to December 2015). Logistic regression was used to obtain ORs related to the proportion of patients who received each of the individual care processes at a site level. For the combined scores, we used multilevel analysis, with the levels defined as patient and hospital, to adjust for correlations between patients within hospitals. A secondary analysis was performed comparing the gap scores, and a sensitivity analysis was also performed to describe the differences between the baseline period (January 2012 to June 2013) and postintervention period (October to December 2014).

Descriptive statistics including $\mathrm{X}^{2}$ tests for comparisons of proportions between the different evaluation periods were used. Level of significance was set at $\mathrm{p}<0.05$.

\section{RESULTS}

Each invited hospital agreed to participate. Overall, 1298 episodes of care from the two pilot sites were included in the analysis ( $p r e=419$; post $=438$ and sustainability $=432$ ). Both pilot sites had a stroke unit which included co-located beds within a geographically defined ward staffed by a dedicated, multidisciplinary team with expertise in stroke. Site 1 was a 400-bed hospital and a regional hub for three small referring rural hospitals (catchment population 400 000). Site 2 was a 530-bed regional hospital providing services for a population of approximately 240000 people, as well as tertiary-referral services for another 670000 . Site 1 admits approximately 450 patients with stroke and TIA per annum and site 2 admits approximately 250 . Both offer a $24 / 7$ thrombolysis service. Site 1 has a rapid-access TIA service staffed by a multidisciplinary team which can provide early rehabilitation. Both pilot hospitals have access to subacute and inpatient rehabilitation services. These hospitals are typical of most hospitals in Queensland contributing data to AuSCR.

Table 1 provides a summary of identified modifiable barriers that were common to both pilot hospitals, broadly described based on need for evidence, the context of care or where facilitation might be required. Common themes included habituation of practice and awareness of practice gaps. More specifically, inconsistent use of existing tools and systems, lack of pharmacist involvement as part of interdisciplinary care, inconsistent procedural knowledge associated with discharge planning, such as clinicians making assumptions about patient eligibility independent of other evidence, and suboptimal recording of data in the AuSCR and medical records were consistent areas identified for improvement. Importantly, it was recognised that existing processes and administrative systems could 


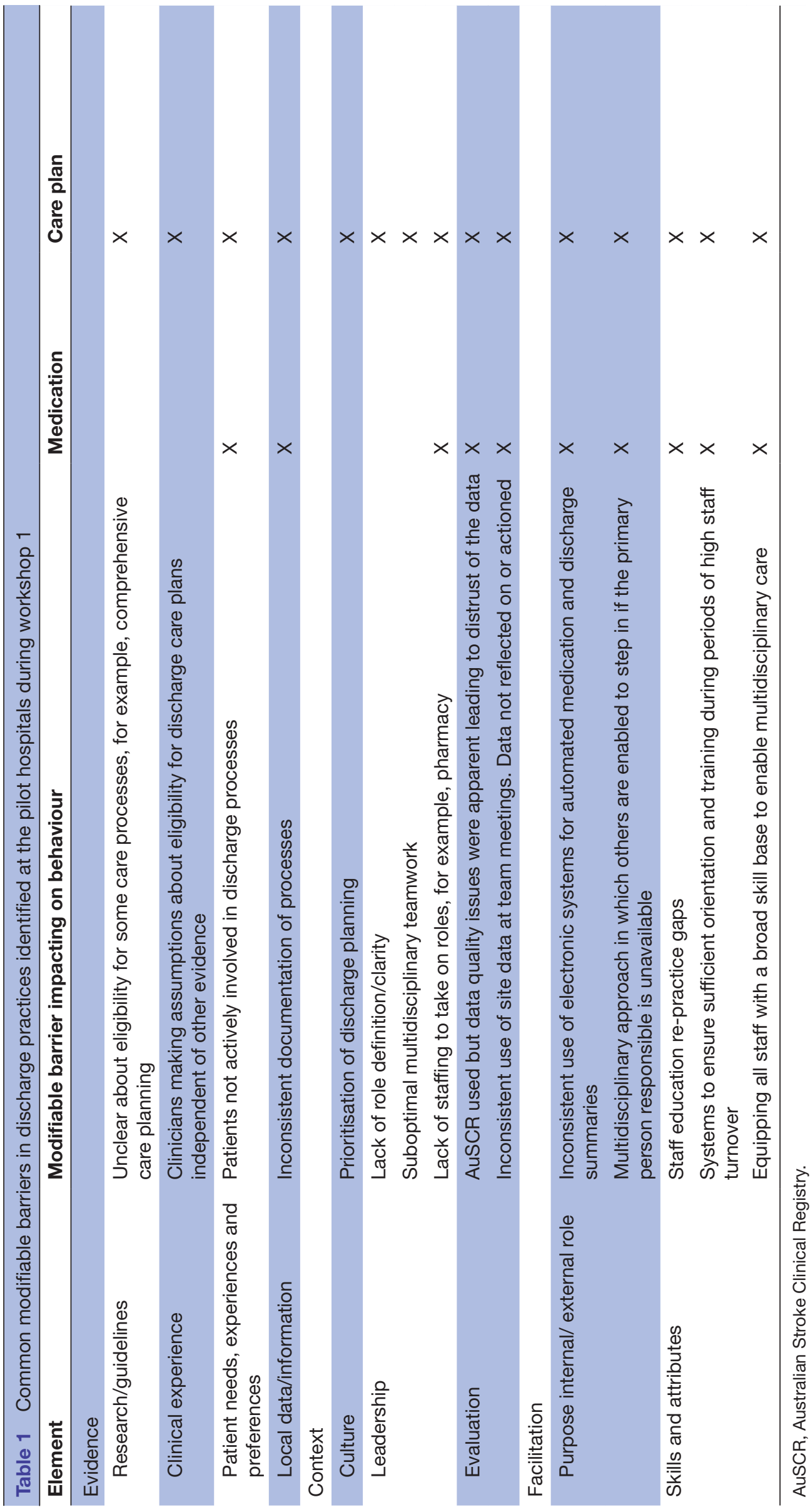

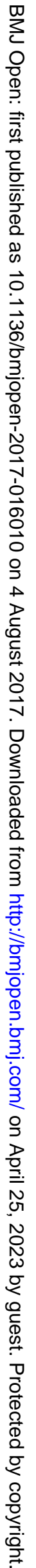


be leveraged by changing workflow processes. Common strategies included providing an example of a comprehensive discharge care plan for clinicians to refer to, using reminders (such as stickers in medical records) to facilitate prescription of medications, and regular reviews of data at team meetings. The health professionals also planned to empower patients and their families to use the My Stroke Journey ${ }^{8}$ pack that was to be provided at time of admission to promote more effective and collaborative discharge care planning. There was recognition that when such interactions with patients and families occurred, this needed to be documented.

\section{Intervention effectiveness}

Patient characteristics were generally similar from the participating hospitals relative to the non-participating hospitals during the preintervention and postintervention periods (online supplementary table III). Some exceptions were ability to walk on admission (site 2: pre $25 \%$, post $75 \%, \mathrm{p}<0.001$ site 1 : pre $50 \%$, post $35 \%$, $\mathrm{p}=0.04$ ). The median age preintervention was 76 (Quandrant (Q)1 25th percentile: 67, Q3 75th percentile: 84) at site 1, 66 at site 2 (Q1: 58, Q3: 77) and 73 (Q1: 61, Q3:81) for the other Queensland sites combined. The proportion of women in the preintervention period was $43 \%$ for site 1, $44 \%$ for site $2 \%$ and $42 \%$ for non-participating sites, and the proportion of ischaemic stroke were $71 \%$ for site $1,67 \%$ for site $2 \%$ and $68 \%$ for non-participating sites. There were some differences in discharge destinations with site 1 commencing initial subacute care within the stroke unit in approximately one-third of patients compared with none at site $2 \%$, and $7 \%$ of non-participating sites.

Overall, the postintervention composite outcome $(89 \%)$ significantly improved compared with the preintervention result from pilot sites $(73 \% ; \mathrm{p}<0.001)$. This was mainly attributable to greater provision of discharge care plans which had the greatest scope for improvement (72\% preintervention vs $94 \%$ postintervention) (table 2 ). Sustainability was achieved for all but one care process (prescribed antiplatelet therapy at discharge) which had a significant $7 \%$ reduction in adherence at 12 months after the intervention. A small non-significant reduction of $4 \%$ in the composite outcome occurred at 12 months (89\% postintervention vs $85 \%$ at 12 months) representing the decay effect. Significant overall improvements were also achieved when the overall benchmark-derived gap scores were compared for the composite outcome (preintervention composite gap score $85 \%$ vs postintervention $94 \% \mathrm{p}<0.001)$ and maintained during the 12-month sustainability period. Other results on the changes in different gaps scores are provided in online supplementary table IV.

Despite overall improvements in the composite outcome and individual care processes, there were variations in the magnitude of improved performance between the pilot sites following the STELAR intervention (table 2). Results from our sensitivity analyses comparing the baseline (hospital selection period) composite outcome and postintervention outcomes showed much greater improvements than comparisons between the preintervention and postintervention periods, and in contrast to the non-participating hospitals (site 1, baseline composite outcome $37 \%$; postintervention $86 \%, \mathrm{p}<0.001$ and site 2 , baseline composite outcome $44 \%$; postintervention $93 \%$, $\mathrm{p}<0.001$ ) (figure 2 and online supplementary figure IV).

\section{DISCUSSION}

Changing clinician behaviour to improve patient care will ultimately lead to better patient outcomes. ${ }^{10} 192428$ The results from this project provide promising data on a new, theory and evidence-informed, multifaceted organisational intervention for improving discharge care after hospitalisation for acute stroke. Overall, significant and sustained improvements in adherence to the discharge processes were demonstrated following implementation of the STELAR programme. The net change in the composite outcome increased $16 \%$ after implementation with a non-significant decay effect at 12-month postimplementation (12\% improvement compared with the preimplementation result or $4 \%$ decay effect). This case study in stroke is relevant to other neurological chronic conditions and provides information to support clinicians in how to design or deliver an organisational intervention that can be tailored to the local context.

The final design of the intervention included several stages since knowledge translation for healthcare professionals is more likely to be successful if stakeholders are involved in the process; readiness for change is achieved and the approach is informed by an assessment of the likely barriers and facilitators. ${ }^{10}{ }^{29}$ Each pilot site had a change champion appointed, and we also used a local opinion leader to deliver the educational component of the face-to-face workshop. This is because local opinion leaders can influence rapid uptake of evidence. ${ }^{18}$ Similar to other authors, ${ }^{30}$ we found mapping the reported barriers and enablers to the $\mathrm{TDF}^{18}$ and corresponding behaviour change techniques ${ }^{17}$ to be a comprehensive approach to support targets for inclusion in an effective intervention. A particularly novel aspect was using experience from 'exemplar hospitals' to inform selection of strategies for inclusion in action plans.

The results are encouraging and accord with the most promising effect sizes for similar types of interventions reported in systematic reviews. ${ }^{10} 24$ If this intervention is considered a form of 'audit and feedback' methodology, then the feedback component we provided was consistent with the literature on the most effective methods for achieving a large effect. Feedback may be more effective when baseline performance is low; the provider of the feedback is a supervisor or a colleague; the data on performance are provided more than once and in verbal and written formats; and when it includes explicit targets and an action plan. ${ }^{19}$ Each of these elements was used in STELAR and provides further evidence that an absolute 


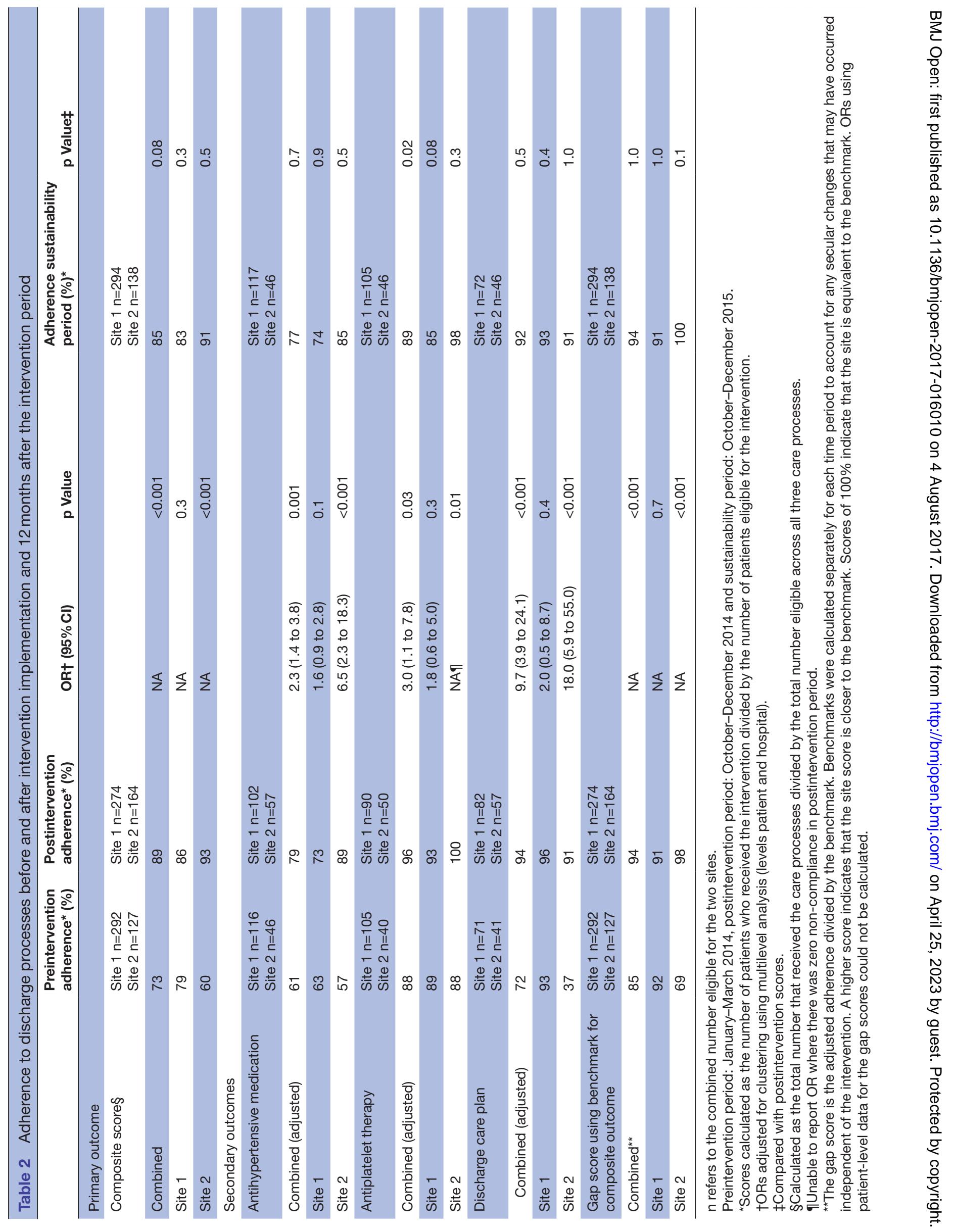




\section{口Pre-STELAR $⿴$ Post-STELAR 12 months post}

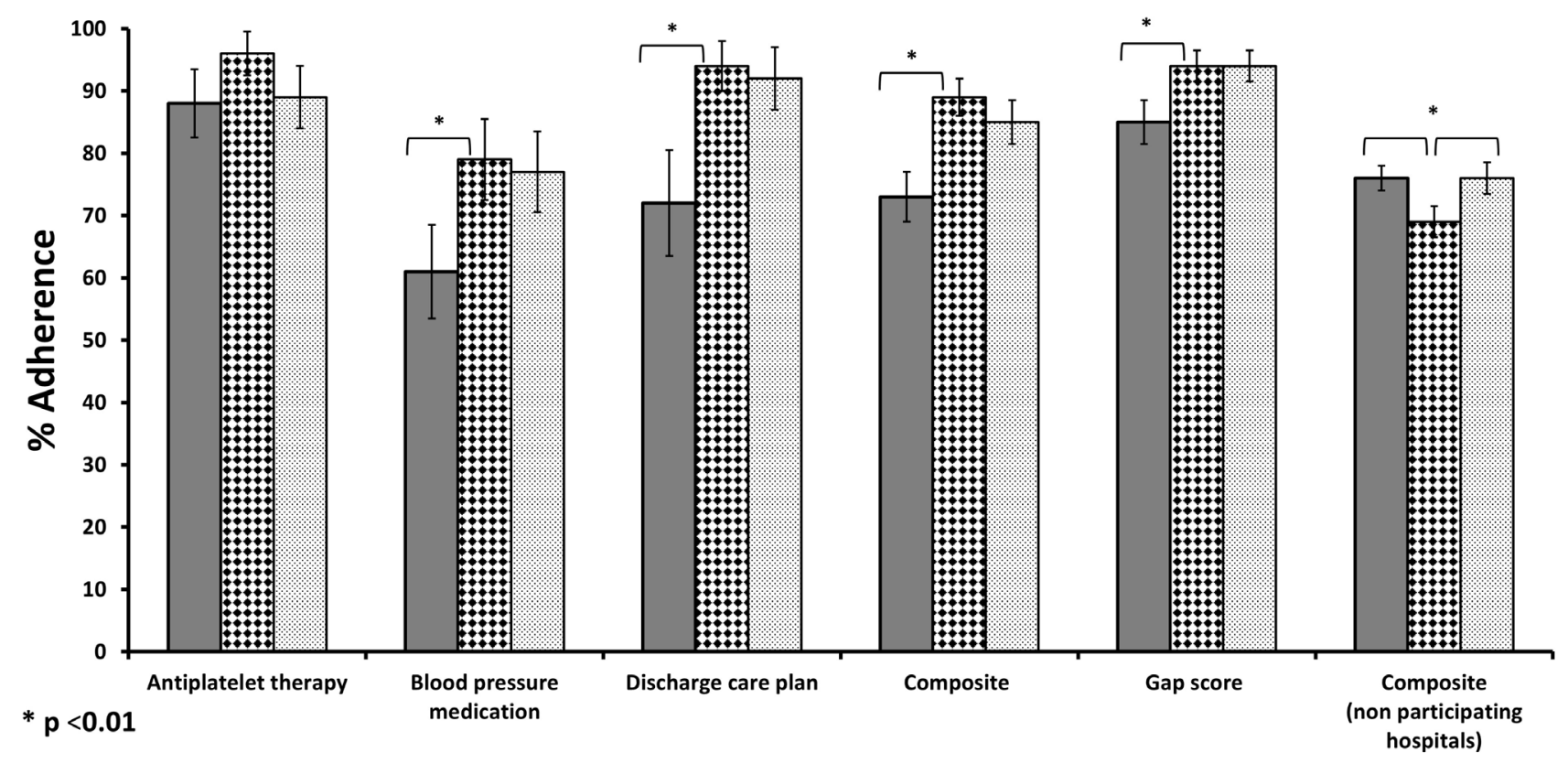

Figure 2 Overall changes in adherence across different time periods for the pilot sites and non-participation hospitals. STELAR, shared team efforts leading to adherence results.

risk difference of $16 \%$, consistent with the reported upper IQR from the included studies in the Ivers and colleagues' review, ${ }^{19}$ is reproducible.

Strengths of our study include use of routinely collected and standardised data available from an established national clinical quality registry, the AuSCR, which facilitates routine and prospective monitoring and quality improvement. $^{31}$ Importantly, we also incorporated reporting of sustainability, the new frontier in quality improvement. ${ }^{11} 12$ The programme was informed by the literature including relevant theoretical frameworks and systematic reviews of effective strategies to achieve clinician behaviour change. Each of the theoretical frameworks we used included the common elements of performance review against the evidence; and barrier management that takes into account local context or attempts to assess or account for the level of adoption. ${ }^{9} 3233$ Previous authors have summarised the factors needed for successful knowledge translation, which include preparing for change, capacity for implementation including people and setting, resources, leverage, desirable implementation enabling features and achieving sustainability. ${ }^{34}$ Importantly, our design provided the possibility to align intervention components with the transtheoretical model of behaviour change: precontemplation (site identification-awareness raising), contemplation (recognising the gaps and considering possible causes (workshop 1)), preparing for action (action plan and change champion appointed), action (action plan implementation with external support) and maintenance (sustainability review) of change. ${ }^{22}$
Limitations included the potential for bias associated with observational studies, including reporting and response bias. Despite strong engagement with, and uptake of, the intervention at both sites, variability in the degree of change in adherence to the care processes at each site postintervention was noted (net change in composite outcome site 1: $7 \%$ and site 2: 33\%). This may have been partly due to ceiling effects for some of the processes and the time delay between enrolment (when sites were made aware of potential for underperformance) and commencement of the interventions. This meant that sites were aware of the project 2 months prior to the start of the baseline data collection period and 9 months prior to the commencement of the intervention. Both sites had improved adherence between the baseline (hospital selection) and preintervention phase (net change in composite outcome site 1: $42 \%$ and site 2: 16\%). Although we did not openly disclose if the hospital was an exemplar or pilot site at the time of the initial invitation, all could access online summary performance reports from the AuSCR at any time. The invitation may have prompted them to review their data. Other external factors such as presentation of data at the annual Queensland Stroke Clinical Network Forum in November 2013 may also have motivated efforts to improve discharge care, as the benchmark thresholds increased over time. If 'awareness raising' (invitation at baseline period) had been accounted for as part of the intervention, the overall results would have been more pronounced as demonstrated in the sensitivity 
analysis (both sites shifted 49 percentage points from baseline (invitation) to postintervention). Encouragingly, further improvements occurred and by the end of the study, both pilot sites were among the top-performing hospitals in Queensland for the measured care processes. We also acknowledge that the low baseline scores for the intervention pilot sites may have resulted in some regression towards the mean in our results. However, the magnitude of change in our pre-scores and post-scores was large and so would have been unlikely to have impacted on the significance of our results. We also acknowledge that type 1 errors from testing of multiple associations or outcomes may be possible, but given our large effect sizes $(>2)$ and our small p-values, type 1 errors in our primary outcome, change in the combined composite score, are unlikely. ${ }^{35}$

In the area of stroke and other neurological conditions, few examples of interventions designed to effectively change clinician behaviour exist. These include multifaceted interventions such as the Get-WithThe-Guidelines-Stroke programme ${ }^{3637}$ and the Quality in Acute Stroke Care Trial that was a nurse-led intervention to improve management of fever, sugar and swallowing in acute stroke units through use of protocols. ${ }^{38}$ Similarly, there is a paucity of evidence for the hospital setting on the sustainability of practice improvement programmes. In a recent scoping review, only seven studies of programme sustainability had been conducted within a hospital environment. ${ }^{11}$ In our future planned work, it will be important to determine if this proposed STELAR intervention is reproducible in more hospitals, and can be extended to other care processes. We are currently undertaking a step-wedge designed study among 12 hospitals located in Victoria (Australia) where this type of intervention has not previously been used following funding obtained from the Ian Potter Foundation in 2016.

In conclusion, improving discharge care in hospitals through a staged, evidence-based, multifaceted intervention was effective and sustainable, and further testing of the intervention in more hospitals would be worthwhile. Importantly, we drew on the cumulative experience from the growing field of knowledge translation to design a programme for stroke, where little previous research has been undertaken. This research is relevant to other clinical domains, where variation in practice for discharge care is widespread.

\footnotetext{
Author affiliations

${ }^{1}$ Stroke and Ageing Research, Department of Medicine, School of Clinical Sciences at Monash Health, Monash University, Clayton, Australia

${ }^{2}$ Stroke Division, Florey Institute of Neuroscience and Mental Health, University of Melbourne, Heidelberg, Australia

${ }^{3}$ Queensland Cerebral Palsy and Rehabilitation Research Centre (QCPRRC),

University of Queensland, Brisbane, Australia

${ }^{4}$ Stroke Foundation, Melbourne, Australia

${ }^{5}$ Nursing Research Institute, St Vincent's Health Australia (Sydney) and Australian Catholic University, Sydney, Australia

${ }^{6}$ School of Nursing, Midwifery \& Paramedicine, University of the Sunshine Coast, Sunshine Coast, Australia

${ }^{7}$ Acute Stroke Unit, The Townsville Hospital, Townsville, Australia
}

${ }^{8}$ Redcliffe Hospital, Redcliffe, Australia

${ }^{9}$ School of Medicine, University of Tasmania, Hobart, Australia

${ }^{10}$ Sunshine Coast Clinical School, The University of Queensland, Nambour, Australia

${ }^{11}$ Queensland Department of Health, Brisbane, Australia

Contributors DAC: conceptualisation and design of the study, drafting of the manuscript and interpretation of the data. NEA: drafting of the manuscript, literature review, analysis of data and interpretation of the data. ESS: revisions to the manuscript, contribution to literature reviews, study coordination and interpretation of the data. KH: revisions to the manuscript, contribution to intervention design and methods, and interpretation of the data. SM: revisions of the manuscript, contribution to intervention design and methods, and interpretation of the data. EH: consumer representative, revision of the manuscript, contribution to intervention design and methods, and interpretation of the data. IM: revisions to the manuscript, contribution to intervention delivery and interpretation of the data. SK: revisions to the manuscript, contribution to focus group and interpretation of the data. MRN: revision of the manuscript, contribution to intervention design and interpretation of the data. RG: revisions to the manuscript, contribution to intervention design and methods, and interpretation of the data.

Funding The Nancy \& Vic Allen Stroke Prevention Fund supported this project which was also supplemented by grant funds from the National Health and Medical Research Council (NHMRC; 1034415). DAC (1063761 c0-funded Heart Foundation) and NEA (1072053) received Research Fellowship support from the NHMRC.

Competing interests None declared.

Ethics approval Metro North Hospital and Health Service, The Prince Charles Hospital, Human Research Ethics Committee (HREC/13/QPCH/279, November 2013).

Provenance and peer review Not commissioned; externally peer reviewed.

Data sharing statement Unpublished programme evaluation data from this study may be available for mutually agreed use by contacting the corresponding author.

Open Access This is an Open Access article distributed in accordance with the Creative Commons Attribution Non Commercial (CC BY-NC 4.0) license, which permits others to distribute, remix, adapt, build upon this work non-commercially, and license their derivative works on different terms, provided the original work is properly cited and the use is non-commercial. See: http://creativecommons.org/ licenses/by-nc/4.0/

C Article author(s) (or their employer(s) unless otherwise stated in the text of the article) 2017. All rights reserved. No commercial use is permitted unless otherwise expressly granted.

\section{REFERENCES}

1. Chin $\mathrm{JH}$, Vora $\mathrm{N}$. The global burden of neurologic diseases. Neurology 2014;83:349-51.

2. Mohan KM, Wolfe CD, Rudd AG, et al. Risk and cumulative risk of stroke recurrence: a systematic review and meta-analysis. Stroke 2011;42:1489-94.

3. Thrift AG, Kim J, Douzmanian V, et al. Discharge is a critical time to influence 10-year use of secondary prevention therapies for stroke. Stroke 2014;45:539-44.

4. Andrew NE, Kilkenny M, Naylor R, et al. Understanding longterm unmet needs in Australian survivors of stroke. Int J Stroke 2014;9(Suppl A100):106-12.

5. Goncalves-Bradley DC, Lannin NA, Clemson LM, et al. Discharge planning from hospital. The Cochrane database of systematic reviews 2016;1:CD000313.

6. National Stroke Foundation. 2015. National Stroke Audit - Acute Services Report. Melbourne, Australia.

7. Cadilhac D, Lannin N, Anderson C, et al. The Australian Stroke Clinical Registry Annual Report 2014: the Florey Institute of Neuroscience and Mental Health. 65, 2015.

8. National Stroke Foundation. My Stroke Journey Melbourne, Australia. 2016. https://strokefoundation.com.au/what-we-do/supportprograms/my-stroke-journey

9. Taylor MJ, McNicholas C, Nicolay C, et al. Systematic review of the application of the plan-do-study-act method to improve quality in healthcare. BMJ Qual Saf 2014;23:290-8.

10. Grimshaw JM, Eccles MP, Lavis JN, et al. Knowledge translation of research findings. Implement Sci 2012;7:50.

11. Francis L, Dunt D, Cadilhac DA. How is the sustainability of chronic disease health programmes empirically measured in hospital 
and related healthcare services?-a scoping review. BMJ Open 2016;6:e010944.

12. Whelan J, Love P, Pettman T, et al. Cochrane update: predicting sustainability of intervention effects in public health evidence: identifying key elements to provide guidance. J Public Health 2014;36:347-51.

13. Tricco AC, Ashoor HM, Cardoso R, et al. Sustainability of knowledge translation interventions in healthcare decision-making: a scoping review. Implement Sci 2016;11:55.

14. Ogrinc G, Davies L, Goodman D, et al. SQUIRE 2.0 (Standards for QUality Improvement Reporting Excellence): revised publication guidelines from a detailed consensus process. BMJ quality \& safety 2015.

15. Squires JE, Sullivan K, Eccles MP, et al. Are multifaceted interventions more effective than single-component interventions in changing health-care professionals' behaviours? An overview of systematic reviews. Implement Sci 2014;9:152.

16. Dehar M-A, Casswell S, Duignan P. Formative and process evaluation of Health Promotion and Disease Prevention Programs. Eval Rev 1993;17:204-20.

17. Michie S, Richardson M, Johnston M, et al. The behavior change technique taxonomy (v1) of 93 hierarchically clustered techniques: building an international consensus for the reporting of behavior change interventions. Ann Behav Med 2013;46:81-95.

18. Cane J, O'Connor D, Michie S. Validation of the theoretical domains framework for use in behaviour change and implementation research. Implement Sci 2012;7:1-17.

19. Ivers N, Jamtvedt G, Flottorp S, et al. Audit and feedback: effects on professional practice and healthcare outcomes. Cochrane Database Syst Rev 2012:CD000259.

20. National Collaborating Centre for methods and tools. Ottawa Model of Research Use: a Framework for adopting innovations. Hamilton: McMaster University, 2010.

21. Rycroft-Malone J. The PARIHS framework--a framework for guiding the implementation of evidence-based practice. J Nurs Care Qual 2004;19:297-304.

22. Prochaska JO, DiClemente CC. Stages and processes of self-change of smoking: toward an integrative model of change. J Consult Clin Psychol 1983;51:390-5.

23. Michie S, van Stralen MM, West R. The behaviour change wheel: a new method for characterising and designing behaviour change interventions. Implement Sci 2011;6:42.

24. Grimshaw JM, Thomas RE, MacLennan G, et al. Effectiveness and efficiency of guideline dissemination and implementation strategies. Health Technol Assess 2004;8:1.
25. Cadilhac DA, Lannin NA, Anderson CS, et al. Protocol and pilot data for establishing the Australian Stroke Clinical Registry. Int J Stroke 2010;5:217-26.

26. Weissman NW, Allison JJ, Kiefe $\mathrm{Cl}$, et al. Achievable benchmarks of care: the ABCs of benchmarking. J Eval Clin Pract 1999;5:269-81.

27. Hall RE, Khan F, Bayley MT, et al. Benchmarks for acute stroke care delivery. Int J Qual Health Care 2013;25:710-8.

28. Flodgren G, Parmelli E, Doumit G, et al. Local opinion leaders: effects on professional practice and health care outcomes. Cochrane Database Syst Rev 2011;8:CD000125.

29. Fitzgerald A, Ogrin R, Hayes K, et al. Balancing Yin and Yang: the development of a framework using participatory action research for the translation and implementation (part 1) of new practices. Asia Pac J 2016;11:14-24.

30. Lipworth W, Taylor N, Braithwaite J. Can the theoretical domains framework account for the implementation of clinical quality interventions? BMC Health Serv Res 2013;13:530.

31. Cadilhac DA, Kim J, Lannin NA, et al. National stroke registries for monitoring and improving the quality of hospital care: a systematic review. Int J Stroke 2016;11:28-40.

32. Kitson AL, Rycroft-Malone J, Harvey G, et al. Evaluating the successful implementation of evidence into practice using the PARiHS framework: theoretical and practical challenges. Implement Sci 2008;3:1.

33. Graham ID, Logan J. Innovations in knowledge transfer and continuity of care. Can J Nurs Res 2004;36:89-103.

34. Braithwaite J, Marks D, Taylor N. Harnessing implementation science to improve care quality and patient safety: a systematic review of targeted literature. Int J Qual Health Care 2014;26:321-9.

35. Feise RJ. Do multiple outcome measures require $p$-value adjustment? BMC Med Res Methodol 2002;2:8.

36. Schwamm LH, Fonarow GC, Reeves MJ, et al. Get With the Guidelines-Stroke is associated with sustained improvement in care for patients hospitalized with acute stroke or transient ischemic attack. Circulation 2009;119:107-15.

37. LaBresh KA, Reeves MJ, Frankel MR, et al. Hospital treatment of patients with ischemic stroke or transient ischemic attack using the "Get With The Guidelines" program. Arch Intern Med 2008;168:411-7.

38. Middleton S, McElduff $P$, Ward J, et al. Implementation of evidencebased treatment protocols to manage fever, hyperglycaemia, and swallowing dysfunction in acute stroke (QASC): a cluster randomised controlled trial. Lancet 2011;378:1699-706.

39. Middleton S, Lydtin A, Comerford D, et al. From QASC to QASCIP: successful Australian translational scale-up and spread of a proven intervention in acute stroke using a prospective pre-test/post-test study design. BMJ Open 2016;6:e011568. 